\title{
How to Define Games and Why We Need to
}

\author{
Jonne Arjoranta ${ }^{1}$
}

Received: 28 February 2019 / Accepted: 20 May 2019 / Published online: 29 May 2019

(c) The Author(s) 2019

\begin{abstract}
This article provides guidelines on how to make useful game definitions and discusses when that is a worthwhile undertaking. It examines a recent article attempting to define videogames (Bergonse in Comput Games J 6(4):239-255, 2017. https ://doi.org/10.1007/s40869-017-0045-4), and uses it as an example to discuss game definitions in general. It concludes with reasons why making a final definition of games is not possible and why we need to continue to define games.
\end{abstract}

Keywords Definition · Game · Videogame

\section{Introduction}

A recent article in The Computer Games Journal (Bergonse 2017: 239, called Fifty Years On) made a remarkable claim:

During the last five decades, videogames evolved into a major component of popular culture as well as a multi-billion-dollar industry. The medium diversified tremendously, currently encompassing simple implementations of numeric games on the screen of a cell phone as well as vast, persistent online worlds on last generation consoles and PCs. In spite of its cultural and economic relevance, few attempts have been made to define what a videogame exactly is. (emphasis added)

Based on this perceived lack of previous definitions, the article then endeavours to give an essentialist definition of videogames, identifying five essential properties. These are summarised in the following definition:

[a videogame is] a mode of interaction between a player, a machine with an electronic visual display, and possibly other players, that is mediated by a meaningful fictional context, and sustained by an emotional attachment

Jonne Arjoranta

jonne.arjoranta@jyu.fi

1 Department of Music, Art and Culture Studies, University of Jyväskylä, PL 35,

40014 Jyväskylä, Finland 
between the player and the outcomes of her actions within this fictional context (Bergonse 2017: 253).

There is one central problem with this: the claim that there is a dearth of previous definitions is demonstrably false (e.g. Bogost 2009; Deterding 2013; Esposito 2005; Karhulahti 2015b; Tavinor 2008; Whitton 2009; Wolf 2001). Because the author bases their argument on that false premise, they fail to take into account almost all of the previous work on this subject. This leads to other problems, as Fifty Years On stumbles over hurdles that others have already overcome. For example, the definition excludes zero-player games (Björk and Juul 2012) and games that do not require "electronic visual displays," like the Dark Room Sex Game (Korsgaard et al. 2008; see also Karhulahti 2015b) or games in the Audio Game Hub (Sonnar Interactive Ltd 2016). Perhaps there are good reasons not to include these in videogames, but it is hard to tell why, when no reasons are given.

It is understandable how one might conclude that there are a lack of videogame definitions, if one only looks at articles in The Computer Games Journal, the journal in which Fifty Years On was published. It is not a journal known for its philosophical discussions. Fortunately, there are other journals that are more likely to publish philosophical articles, and there are book chapters, conference articles and books about the subject. It might make sense to publish this response in one of these places, but, considering that the article that this is a response to was published in The Computer Games Journal, it seems sensible to respond in the same journal.

The goal here is not to focus on Fifty Years On. It is used here as an example to discuss the problems of definitions in general, because it happens to be a good example of a mistaken approach to game definitions. However, the article is not alone in making that mistake: struggling with game definitions seems to be a persistent feature of game studies. This is perhaps understandable: game studies is a multidisciplinary field and many disciplines do not have long traditions of theorizing definitions. Because of this, it might not be entirely clear for all researchers what the purpose of defining things is and what kind of activity making definitions is. Hopefully, reading this article will clarify some of that confusion.

Fifty Years On discusses videogames. As the author notes, there are alternative terms that could be used, like computer game or console game (Bergonse 2017: 241; cf. Karhulahti 2015b). I will mostly use the word 'game'; this should be read to include what Fifty Years On discusses as videogames. This is not because I think that videogames and games are the same thing (but they are related, Bergonse 2017: 240; cf. Karhulahti 2015a), but because the process of defining both is similar and benefits from the same considerations. Games and videogames may not be the same thing, but the definitions of both are definitions, and therefore understanding definitions helps in making definitions for both. The problems in the definition literature of both also tend to be similar, so discussing them together is useful in clarifying issues that appear in relation to both. Regardless of which one you are trying to define, this paper points out potential pitfalls and suggests solutions.

In this article, first, I discuss the alleged bias in game studies. Second, I show that there are many previous definitions of games and videogames. Third, I discuss what definitions are and what they are useful for. Fourth, I draw upon previously published 
literature to show how to make useful (video)game definitions. Fifth, I look at when and why making definitions is useful in the first place. If there is already a rich history of previous definitions, is it useful to keep defining games? I argue that it is, and by doing so highlight a problem with Fifty Years On: that it argues for a essentialist definition for a concept that is highly contextual. There is a good reason to keep defining games despite all the previous definitions, but that reason has more to do with definitions than with games. That is why I end with a discussion on why using an essentialist definition for a cultural phenomenon is not the best match.

\section{Is There a Bias in Game Studies?}

Many of the definitions of games include videogames in games because the definitions have been formulated after videogames became a major cultural force and a creative industry. People making these definitions are interested in defining games because of the rise of videogames. Fifty Years On does not include videogames in games because it claims to identify a bias in game studies publications:

[T]here persists a tendency to approach videogames from a game studies perspective, therefore assuming them to be essentially the latest iteration of games. Independently of the important relations between both concepts, this leads to a lack of concern with specifically defining videogames per se, with considerations being rather centred on videogames vis-à-vis traditional gaming (Tavinor 2008). (Bergonse 2017: 240)

It is entirely possible that game studies could focus too much on games in general and therefore miss what is unique to videogames. However, this seems unlikely, considering the history of exactly the opposite happening, with game scholars focusing on a relatively small set of digital games, with a special focus on the World of Warcraft (Blizzard Entertainment 2004; see Coavoux et al. 2017).

The genesis of game studies as a field is often stated as 2001, when "Computer Game Studies, Year One" (Aarseth 2001) was published and the journal Game Studies started. As the name of the editorial introduction implies, videogames have had a prevalent position in game studies from the beginning of the field. Similarly, scholars have noted that the international association for game scholars is called the Digital Games Research Association, not the more inclusive Games Research Association, despite some members' vocal opposition.

Stenros and Waern (2011: 1) call this overemphasis on digital games the "digital fallacy" after noting that the "field of game studies is currently dominated by the study of digital games"- and this does not seem to be any less true almost a decade later. Therefore it does not seem that game studies suffers from insufficient attention on videogames; the opposite seems more likely. Fortunately, this has lead to the creation of the journal Analog Game Studies, in an effort to provide a place for publishing research on non-digital games. 


\section{Previous Definitions}

A good starting point for looking at previous definitions of games is the systematic literature review by Stenros (2017), which looks at over 60 definitions of games since the 1930s. Not all of them are about videogames (some predate videogames), but if videogames have anything to do with games, looking at how games have been previously defined might be enlightening. One exemplary definition is provided by Suits (1967b, 1980). Suits (1967b: 156) considers that:

to play a game is to engage in activity directed toward bringing about a specific state of affairs, using only means permitted by specific rules, where the means permitted by the rules are more limited in scope than they would be in the absence of the rules, and where the sole reason for accepting such limitation is to make possible such activity.

This highly formal definition covers games, but perhaps it covers too much. Even if one disagrees with the position that videogames are games, and therefore is only interested in definitions of videogames, Fifty Years On is not the first to try to define to videogames. For example, Esposito (2005) presents a very succinct definition:

A videogame is a game which we play thanks to an audiovisual apparatus and which can be based on a story. (emphasis in original)

Perhaps this rather minimalistic definition is lacking and can be added to, but it makes a good starting point for the discussion (for other definitions of videogames see e.g. Bogost 2009; Deterding 2013; Karhulahti 2015b; Tavinor 2008; Whitton 2009; Wolf 2001). For the purposes of the current examination it is sufficient to note that there are previous definitions and making new ones without being in dialogue with that tradition is unlikely to produce anything substantially better. However, the goal of this article is not to find the best definition of videogames, but to discuss how one arrives at a useful definition, and what the purpose of making definitions is in the first place.

\section{What Are Definitions?}

To understand game definitions it helps to first understand what definitions are. ${ }^{1}$ There are different types of definitions, with different goals. Definitions can have, for example, explicative, analytical or persuasive functions (Laas 2017: 84-85). They can be used in different contexts, for example in political arguments. These are all equally valid uses for definitions, but this article focuses on how definitions work in science and philosophy, because the goal is to help game studies deal with game definitions. The following discussion starts by focusing on

\footnotetext{
1 The following section simplifies some issues and ignores others in order to present a short and understandable explanation of what definitions are. For a more thorough introduction to these issues, see Cohen (1934). For a discussion on essentialist definitions, see Walton and Macagno (2009).
} 
essential definitions, before moving on to discuss a Wittgensteinian approach to definitions that better fits our specific case.

An approach that tries to give an essentialist definition for something typically tries to identify the necessary and sufficient conditions that must be fulfilled in order for something to be essentially that thing. 'Necessary' and 'sufficient' are technical terms in philosophy, deriving from logical terminology. Essential definitions succeed by including the kinds of things being defined, and only those things. Conversely, they fail by either not including all of the things that are being defined or including things that are not being defined. As an example, we can think about videogames in the genre of walking simulators. If we include challenge (e.g. Juul 2003) in our definition of games, Proteus (Key and Kanaga 2013) is not a game. We can then either think that the definition fails by not being inclusive enough, or that Proteus is indeed not a game and the definition makes an important distinction between it and proper games.

I will not go into detail here how essential proofs are typically constructed, instead I will focus on why this is usually done. Words like 'essential' might lead one to conclude that a definition that defines the essential properties of something has created some kind of metaphysical act of delimitation, drawing the limits of some object perfectly against the background of the noisy universe. The reality is somewhat more mundane, as definitions are less about recognising perfectly the essence of the objects around us and more about solving practical problems, often caused by language. Using technical tools like necessary and sufficient conditions is useful, because they allow logicians to use tools developed for logical thinking to reason about the relations between things.

In this sense, definitions work like mathematical axioms, taken to be true because they help in making sense of some problem that is otherwise difficult to solve. They have pragmatic consequences, because by assuming certain things to be true they allow us to reason about the reality we live in.

They also have other practical consequences: "knowledge itself is power," as noted by Francis Bacon (see García 2001 on how Bourdieu and Foucault further elaborated on this relationship). Making definitions is exercising power. Definitions affect the terms in which things are discussed: what are acceptable answers to questions and what are not. Experts are in positions of power because of their ability to define the terms of discussion. That is why definitions are not just neutral descriptions of reality and can have consequences beyond their typical academic confines.

One of the reasons definition problems keep coming up in relation to games is because one of the most influential philosophers of the twentieth century, Ludwig Wittgenstein, happened to write about games in his book Philosophical Investigations (2001). He discusses games as an example at the beginning of the book (Wittgenstein 2001 paragraph §3):

It is as if someone were to say: "A game consists in moving objects about on a surface according to certain rules..." -and we replied: You seem to be thinking of board games, but there are others. You can make your definition correct by expressly restricting it to those games. 
While he discusses other things after this, he returns to games a bit later in the book (Wittgenstein 2001 paragraph §66):

Consider for example the proceedings that we call "games". I mean boardgames, card-games, ball-games, Olympic games, and so on. What is common to them all? - Don't say: "There must be something common, or they would not be called 'games"'-but look and see whether there is anything common to all. - For if you look at them you will not see something that is common to all, but similarities, relationships, and a whole series of them at that. To repeat: don't think, but look!

Wittgenstein seems to be saying that if one looks at games, one does not find something "common to" all games, but their relation to each other. He continues this line of thought for a while and finally concludes that:

we see a complicated network of similarities overlapping and criss-crossing:

sometimes overall similarities, sometimes similarities of detail.

It is easy to look at this discussion and conclude that Wittgenstein is claiming that games cannot be defined. It might even lead one on a quest to show how he was wrong by providing a working definition of games (cf. Suits 1980). However, this misunderstanding stems from the misreading that Wittgenstein is here writing about games, when he is in fact concerned with how language works (e.g. Arjoranta 2014; Laas 2017). Especially to the confusion of game scholars, he happens to use games as an example of how language operates. Therefore, the lesson should not be that games cannot be defined, but that language works by a "complicated network of similarities" that Wittgenstein calls family resemblance.

If this is applied to games, it means that they should be understood as a family of related concepts that share family resemblances. ${ }^{2}$ Wittgenstein continues his argument by explaining where these family resemblances come from and by considering some possible counter-arguments, but for the purposes of this discussion it is sufficient to understand his idea of family resemblances and how it informs understanding definitions.

Laas (2017: 84) summarises the Wittgensteinian approach to definitions as four central claims:

(a) definitions are of different kinds;

(b) definitions are relative to the definer's purpose;

(c) definitions do not capture the essences of things; and

(d) the meanings of definitions, like that of concepts and linguistic entities in general, are language-game and theory-dependent.

\footnotetext{
${ }^{2}$ Fifty Years On also recognises that some previous authors (e.g. Feige 2012; see also Arjoranta 2014; Whitton 2009) have used Wittgenstein's (2001) concept of family resemblances to make sense of game definitions, but it curiously identifies this approach as "ignor[ing] the subject" (2017: 240) instead of solving the problem it seems to be struggling with.
} 
Now, with a better understanding of how definitions operate and what they are useful for, we can turn back to thinking about game definitions.

\section{How Can We Make Useful Game Definitions?}

In order to make useful game definitions, it is first necessary to know why we are making them. As shown in the previous section, there are different kinds of definitions and they serve different purposes in relation to the definer's purpose. A new definition should, then, be thought of in relation to a problem one is trying to solve. In this sense, the philosophical task of defining things is also immensely pragmatic.

For example, if one is trying to think about the differences between games and other interactive media, like interactive literature, then thinking about what makes games games and not literature can help in illuminating that situation. The definition provided in that context is unlikely to be very useful for other tasks, like solving whether esports should be thought of as sports. Because definitions are relative to purpose, these two different problems require different solutions.

In addition to usefulness in relation to purpose, there are other criteria that can be used to evaluate definitions (Laas 2017: 90-91):

1. Who has the power to define something? Should they have authority to do so? Should their view be taken for granted? Who is affected by the definition and should their views be taken into account?

2. Can we identify exemplars of the thing we are identifying? Does the definition rule out commonly accepted examples? If so, does it do so for good reasons?

3. What does the definition highlight or emphasise? What does it downplay or ignore? For what reasons?

The above applies to all definitions, not just to definitions of games. In addition to fitting the task at hand, game definitions should also be useful for discussing games and in solving problems related to them. Fortunately, there are a variety of earlier definitions that can either be used as-is, or if the context does not correspond to one of the previously considered, be used as a point of departure for a new definition.

If one is required to make a new definition, it should probably say something useful about games. A typical way to solve this in game studies has been to look at how previous definitions have discussed games, solve any issues with them and provide a synthesis (e.g. Juul 2003). One thorough review of this type is by Stenros (2017), a systematic review of over 60 definitions. Instead of providing a definition, it synthesises the issues game definitions seek to answer into 10 questions or points of contention that a good game definition answers:

1. What are rules?

2. Do games have a function?

3. Are games an artifact or an activity or a muddle of the two?

4. How games exist in relation to the quotidian? 
5. What are players?

6. What do games produce?

7. What is the role of competition?

8. What about goals?

9. What sorts of phenomena are relevant for games?

10. What purpose do definitions serve?

Answering these questions does not guarantee that a game definition is useful, but it does help to make sure that a definition takes previous work in the field into consideration. Taking previous research into consideration is no guarantee of quality, and it is entirely possible to make a useful definition without knowing anything about previous research; however, it is simply more difficult, and prone to run into problems that others have already solved.

This article will not try to provide an exhaustive answer to each and every one of Stenros' (2017) questions in all their possible configurations. Instead, it will continue by discussing why making definitions is necessary in the first place.

\section{Why Define Games?}

For clarity, let us first discuss some cases where many game scholars are often tempted into making definitions when it is not necessary.

1. The first case is whenever you are writing about games. Perhaps because they have seen so many other game scholars give definitions, game scholars often give game definitions when they are studying some specific aspect of games. ${ }^{3}$ This probably hinders whatever argument is being put forward in the article more than it helps, since it stops the flow of the main argument and provides opportunities for disagreement, especially if the definition is poorly made.

2. The second case is whenever another, existing, definition would work. There are multiple previous definitions made by people who have struggled with these questions for a long time. They have published those definitions after intense discussions, sometimes after decades of work, and often after multiple rounds of peer-review (cf. Suits 1967b, 1980). It is possible that you might be able to do better than they have, but probably not before working on the issue for a significant amount of time - which you could instead put into whatever aspect of games you are actually interested in.

You do need a new definition, when

\footnotetext{
${ }^{3}$ As an example, in Arjoranta (2011) I define games as "a form of procedural media: they are systems with certain internal logics." This definition has multiple problems and adds little to understanding game hermeneutics, the actual topic of that paper.
} 
1. The reader is going to be confused about what you mean with 'games' or 'videogames'. In most cases when you are writing about games, the reader is going to recognise the examples you use and agree — at least to the extent that it matterson whether they are games. For example, if you write about the Gothic monsters in Fallout 3 (Bethesda Game Studios 2008; Piittinen 2018), the reader is unlikely to stop reading and ask "but is Fallout 3 a game?" In contrast, if you are going to argue that puzzles are not games (Karhulahti 2013), you might benefit from some definitional work.

2. The act of making the definition is the argument itself. You might want to make a new game definition, if you are working on discussing games in a novel way to highlight some aspects that are otherwise easily ignored or have been previously overlooked. You can also use definitions in this way to explore some aspects of games or other phenomena, by asking "what would happen if we included $\mathrm{X}$ in what we consider to be games?" Is life a game we are playing (Suits 1967a)? You play Capoeira, but is it a game ${ }^{4}$ Are digital board game adaptations board games? A working definition of games can perhaps help with these questions.

3. There is no previous (useful) definition for the context you are considering and making a new definition performs some kind of analytical work that has pragmatic consequences. This differs from the previous case in that you are still discussing something that is considered a game, but from a perspective that has been explored less. Making the assumptions you make about games more explicit can help when performing the rest of the analytical work for your actual research question.

There is another reason why we might need to keep making game definitions. Because it is slightly more complicated than the previous examples discussed, I will discuss it separately and introduce more background in order to understand it. There is a slight change of perspective in the next section relative to the previous sections. While the previous three points relate to the pragmatic and persuasive benefits of definitions (Laas 2017: 85-86), the next section discusses the philosophical aspects of definitions and why we might need to keep making game definitions whether we want to or not.

\section{Why Keep Defining Games?}

Games are a cultural phenomenon. While most animals play, humans have a tendency to make games. The board game Mehen was played in Egypt over 500 years ago (Crist and Vaturi 2008). Mehen had something to do with burial rituals, but its exact purpose and rules have been lost. It would be relatively odd to include it in funeral rituals today, even if one knew the rules. Mehen has not changed, but the culture around it has.

\footnotetext{
${ }^{4}$ Capoeira is a Afro-Brazilian martial art and dance that, according to its practitioners, is played.
} 
If an ancient Egyptian game scholar needed to define games, they might have included something about games being used as part of burial ceremonies. It is unlikely that you would find a contemporary definition that says anything about burials, but many of them include something about fun or enjoyment (e.g. Aarseth 2007). Is our hypothetical Egyptian scholar wrong, since their definition does not fit newer examples? Yes, but no more than Aarseth (2007). Definitions of cultural phenomena are necessarily tied to the cultural context in which they are made. When the cultural context changes - new examples are created, norms change- the definitions need to change too.

In philosophical terminology, our current historical and cultural context is our "horizon" (Gadamer 2004). This is a spatial metaphor: we can only see as far as the horizon, but no further. Our horizon can expand, especially when it comes to understanding history in the light of new information, but ultimately we are always bound to some limits, beyond which we cannot see. The next genre-defining game is always around the corner and the role of play in society is constantly changing (cf. Frissen et al. 2015). However, it would be a mistake to understand this to mean that there is no point in making definitions or that all of them are equally useless. A definition can be better or worse within a particular cultural and historical context and for a particular purpose. The only thing our limited horizon rules out as impossible is coming up with a perfect, eternal definition of all games for all purposes.

There is no final definition of games as long as there are people capable of both playing games and thinking about them. New examples of games or new practices around games need new definitions. This means that game scholars are not going to run out of work anytime soon; the best we can do is to keep updating our knowledge to fit the world in which we live.

\section{Conclusions}

Definitions are tools for analysis or persuasion. They help us understand the world or convince others. They are ways to describe or create reality, not capture it. There is no one, perfect definition for anything, because the quality of a definition is contingent on the purpose of the definition.

Useful game definitions are not confused about the purpose of definitions, they fit the particular use case and take into account previously published work. It is possible to make a useful game definition without being in dialogue with previously published work; it is just very unlikely to succeed.

You may want to define games if the reader is going to be confused by what you mean by games, you argue for a specific way of framing what games are or the definition does the analytical work of no previous definition. There are also philosophical reasons why we need new game definitions: because games are a cultural phenomenon, they keep changing. Even if our games do not change, our perspective on games keeps changing as the culture around them changes. 
Acknowledgements Open access funding provided by University of Jyväskylä (JYU). I wish to thank Michael Debus, Andreas Geisler, Paweł Grabarczyk, Michael Heron, Veli-Matti Karhulahti and the ITU WIP seminar participants for their comments on this article.

Funding Funding was provided by Academy of Finland (Grant No. 312397).

\section{Compliance with Ethical Standards}

Conflict of interest The corresponding author states that there is no conflict of interest.

Open Access This article is distributed under the terms of the Creative Commons Attribution 4.0 International License (http://creativecommons.org/licenses/by/4.0/), which permits unrestricted use, distribution, and reproduction in any medium, provided you give appropriate credit to the original author(s) and the source, provide a link to the Creative Commons license, and indicate if changes were made.

\section{References}

Aarseth, E. (2001). Computer game studies, Year One. Game Studies, 1(1).

Aarseth, E. (2007). I fought the law: Transgressive play and the implied player. In A. Baba (Ed.), Situated play, DiGRA 2007 (pp. 130-133). Tokyo: University of Tokyo.

Arjoranta, J. (2011). Do we need real-time hermeneutics? Structures of meaning in games. In DiGRA'11-Proceedings of the 2011 DiGRA international conference: Think design play (p. 13). Utrecht: Utrecht School of the Arts.

Arjoranta, J. (2014). Game definitions: A Wittgensteinian approach. Game Studies, 14(1), 35.

Bergonse, R. (2017). Fifty Years on, What exactly is a videogame? An essentialistic definitional approach. The Computer Games Journal, 6(4), 239-255. https://doi.org/10.1007/s40869-017-0045-4.

Bethesda Game Studios. (2008). Fallout 3. Rockville, MD: Bethesda Softworks.

Björk, S., \& Juul, J. (2012). Zero-player games. Or: What we talk about when we talk about players. In The philosophy of computer games conference, Madrid.

Bogost, I. (2009). Videogames are a Mess. Ian Bogost. Personal Website.

Coavoux, S., Boutet, M., \& Zabban, V. (2017). What we know about games: A scientometric approach to game studies in the 2000s. Games and Culture, 12(6), 563-584. https://doi.org/10.1177/15554 12016676661.

Cohen, M. F. (1934). An introduction to logic and scientific method. Kansas City: Harcourt, Brace \& World.

Crist, W., \& Vaturi, A. (2008). Board games in antiquity. In H. Selin (Ed.), Encyclopaedia of the history of science, technology, and medicine in non-western cultures (pp. 1-10). Dordrecht: Springer. https ://doi.org/10.1007/978-94-007-3934-5_9836-1.

Deterding, S. (2013). Modes of play: A frame analytic account of video game play. Ph.D. thesis.

Entertainment, Blizzard. (2004). World of warcraft. Irvine, CA: Blizzard Entertainment.

Esposito, N. (2005). A short and simple definition of what a videogame is. In DiGRA 2005 conference: Changing views-worlds in play.

Feige, D. M. (2012). Computer games as works of art. In J. Fromme \& A. Unger (Eds.), Computer games and new media cultures: A handbook of digital games studies (pp. 93-106). Dordrecht: Springer. https://doi.org/10.1007/978-94-007-2777-9_6.

Frissen, V., Lammes, S., de Lange, M., et al. (Eds.). (2015). Playful identities: The ludification of digital media cultures. Amsterdam: Amsterdam University Press.

Gadamer, H.-G. (2004). Truth and Method (2nd ed.). London: Continuum.

García, J. M. R. (2001). Scientia Potestas Est-knowledge is power: Francis Bacon to Michel Foucault. Neohelicon, 28(1), 109-121. https://doi.org/10.1023/A:1011901104984.

Juul, J. (2003). The game, the player, the world: Looking for a heart of gameness. In M. Copier \& J. Raessens (Eds.), Level up: Digital games research (pp. 30-45). Utrecht: Utrecht University.

Karhulahti, V.-M. (2013). Puzzle is not a game! Basic structures of challenge. In Proceedings of DiGRA 2013: DeFragging game studies. 
Karhulahti, V.-M. (2015a). Adventures of Ludom: A videogame geneontology. Dissertation. University of Turku, Turku.

Karhulahti, V.-M. (2015b). Defining the videogame. Game Studies 15(2).

Key, E., \& Kanaga, D. (2013). Proteus. Twisted Tree Games.

Korsgaard, L., Wilson, D., Dimovska, D., et al. (2008). Dark room sex game. Copenhagen: IT University of Copenhagen.

Laas, O. (2017). On game definitions. Journal of the Philosophy of Sport, 44(1), 81-94. https://doi. org/10.1080/00948705.2016.1255556.

Piittinen, S. (2018). Morality in let's play narrations: Moral evaluations of Gothic monsters in gameplay videos of Fallout 3. New Media Society. https://doi.org/10.1177/1461444818779754.

Sonnar Interactive Ltd. (2016). Audio game Hub. Auckland: Sonnar Interactive Ltd.

Stenros, J. (2017). The game definition game: A review. Games and Culture, 12(6), 1-22. https://doi. org/10.1177/1555412016655679.

Stenros, J., \& Waern, A. (2011). Games as activity: Correcting the digital fallacy. In M. Evans (Ed.), Videogame studies: Concepts, cultures and communication. Oxford: Inter-Disciplinary Press.

Suits, B. (1967a). Is life a game we are playing? Ethics, 77(3), 209-213.

Suits, B. (1967b). What is a game? Philosophy of Science, 34(2), 148-156.

Suits, B. (1980). The grasshopper: Games, life and Utopia. Toronto: University of Toronto Press.

Tavinor, G. (2008). Definition of videogames. Contemporary Aesthetics, 6, 16.

Walton, D., \& Macagno, F. (2009). Classification and ambiguity: The role of definition in a conceptual system. Studies in logic, grammar and rhetoric, 16(29), 245-264.

Whitton, N. (2009). Learning with digital games: A practical guide to engaging students in higher education. New York: Routledge.

Wittgenstein, L. (2001). Philosophical investigations: The German text, with a revised English translation (3rd ed.). Oxford: Blackwell.

Wolf, M. J. P. (Ed.). (2001). The medium of the video game. Austin: University of Texas Press. 